\title{
Weight loss has a rapid positive impact on the obese person's attitudes. Attitudes that could disturb weight loss attempts, decrease in number as the successful weight loss gets started
}

\begin{abstract}
This is the second part of the study of attitudes that would disturb weight loss attempts. In the first part of the study the attitudes causing difficulties in weight loss were searched in interviews with former dieters. These results have been published earlier. All together 15 attitude-related factors that disturb the weight loss efforts were found.

In this second part of the study it was studied what would happen to these found difficult attitudes as the successful weight loss period gets going. The dieters $(\mathrm{N}=43)$ were discussed both before and after the dieting period of eight weeks of very low calorie diet and the existence of these difficulties was analyzed during the discussion. As a result the amount of these disturbing factors decreased in number as the weight loss happened. The total amount of disturbing attitude- related factors in this group was 222 at the beginning and 82 at the end of the successful weight loss period. Only basic personal encouragement was given during the weight loss period as a mental support. No psychological intervention was made.
\end{abstract}

Conclusion

These findings support the "Just do it"-approach when it comes to weight loss. Difficulties felt in the beginning of the diet disappeared or decreased in number along with the success in dieting. There can be found difficulties at the starting point of the dieting, their existence can be analyzed but they do not need immediate attention for the dieting period to be successful.

Keywords: weight loss management, management of obesity, factors in weight loss
Volume 4 Issue 5 - 2016

Heidi Luotolahti,' Ilkka Kantola, ${ }^{2}$ Jorma

Viikari' $^{\prime}$

'Department of Medicine, Turku University, Turku, Finland

${ }^{2}$ Department of Medicine, Turku University Hospital, Finland

Correspondence: Heidi Luotolahti, Department of Medicine, Turku University, Turku, Finland, Tel +358407453754, Fax +35826277999, Email heiluo@utu.fi

Received: October 30, 2015 | Published: May 26, 2016
Abbreviations: IN, inertia; CU, culinary attitude; DE, depressive mood; RE, restlessness; $\mathrm{SB}$, superiority attitude; $\mathrm{CR}$, criticism; BI, bitterness; FD, food dependency; DI, experiencing general difficulties in weight loss; IC, impaired physical condition; HG, hunger; ICA, incapacity; PP, peer pressure; LT, lack of time; UN, unrealistic activity; UHT, university hospital of Turku

\section{Introduction}

In the first phase of this study it was tried to find out whether there are some attitude- and/or resources- related factors that could play a role in disturbing the weight loss process. ${ }^{1}$ It was tried to find out whether these factors could be found during a 20-45 min discussion

Table I Factors found during which the weight loss attempt was analyzed in a conversational style. The aim was also to know which of the factors would be statistically most significant. We found 15 attitude-related factors and the most significant of them seemed to be related to depressive mood, food dependence, restlessness and bitterness (Table 1).

In this second phase it was studied what happens to these factors as the successful weight loss starts to happen. It was studied whether "Just do it"-approach is realistic or not in weight loss attempts or would it be better first to concentrate more in the analysis of the difficulties experienced in previous weight loss attempts and maybe provide with some professional psychological help first. Little is known about the changes in these attitudes during weight loss period and we felt it would be useful to do that.

\begin{tabular}{ll}
$\begin{array}{l}\text { Name of } \\
\text { the factor }\end{array}$ & A short description of the factor \\
\hline IN & $\begin{array}{l}\text { Laziness or lack of activity was felt. "Too much trouble", neutral mood, but enthusiasm is lacking. The subject expressed that dieting and } \\
\text { weight loss efforts in general were too demanding."I do not have the energy now."' I do not want to be that meticulous about food." } \\
\text { Too much attention is needed to monitor my eating habits."' Too much of a fuss!" }\end{array}$ \\
CU & $\begin{array}{l}\text { Life was centered on the food in a manner that disturbed the weight loss process. Food was an important part of life. } \\
\text { The subject told himself/herself about the low mood. The depressive mood was considered to be present only, when the subject } \\
\text { admitted it. }\end{array}$
\end{tabular}


Table Continued....

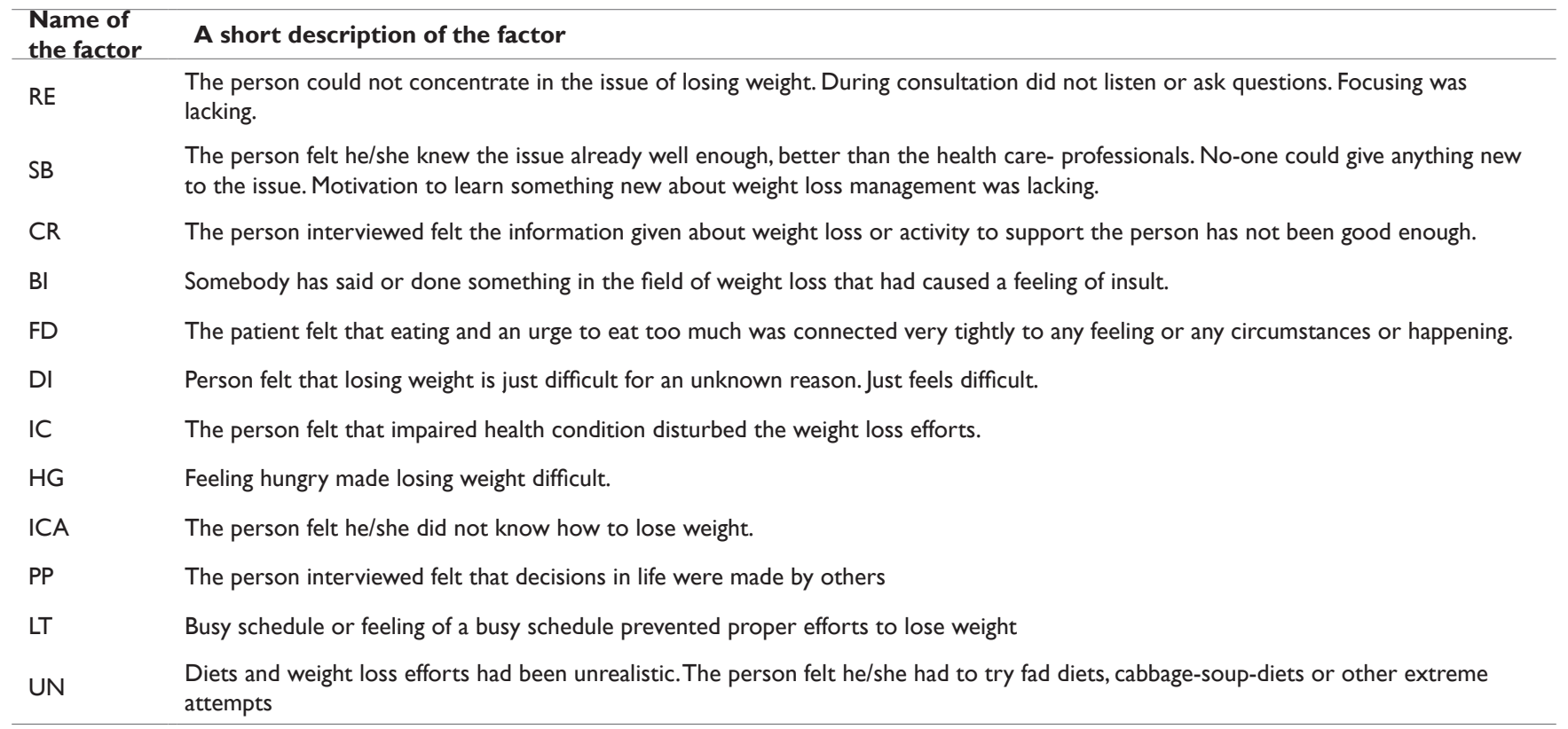

\section{Methods}

The group of 43 obese people started the Very Low Calorie diet (VLCD) period of eight weeks as outpatients in the University Hospital of Turku, during which they successfully lost weight. They were interviewed before and after the diet and the amount of factors present at the start of the diet period and immediately after the successful diet period were observed and counted and the statistical significance of the change in the amounts was counted. The interviewing process happened the same way as in our previous study with the exception that all these interviews took place personally, not by phone. The same interviewer did both discussions: the discussion before the diet period and the discussion after a successful period.

The study group in the second phase consisted of 43 persons who took part in the eight week of VLCD course successfully in the University Hospital of Turku (UHT). The mean age was 44.7 7.0years, female $27 / 62 \%$, male $16 / 38 \%$. Their weight was at the start of the VLCD -course $139.3 \pm 5.9 \mathrm{~kg}$, BMI $47.3 \pm 12.3 \mathrm{~kg} / \mathrm{m}^{2}$ and after the eight week course their weights were $125.5 \pm 2.3 \mathrm{~kg}$, BMI $42.7 \pm 6.4 \mathrm{~kg} / \mathrm{m}^{2}$ and the weight lost was $13.8 \pm 5.9 \mathrm{~kg}$. Only successful dieters were included in this study. No psychological intervention took place during the VLCD-period.

\section{Results}

On our study we found out, that the attitudes disturbing weight loss that we found in our previous study decrease in number as the successful weight loss happens (Figure 1) (Table 2). The total amount of factors decreased statistically extremely significantly as the successful weight loss happened (Table 3 ). The statistically significant changes happened in the inertia, culinary, restlessness and lack of time- factors even as sole factors (Table 4).

Table 2 Appearance of the factors before and after the eight week VLCD

\begin{tabular}{lllll}
\hline Factor & Before VLCD $\mathbf{n}=\mathbf{4 3}$ & Before VLCD percent & After VLCD $\mathbf{n}=\mathbf{4 3}$ & After VLCD per cent \\
\hline IN & 17 & $40 \%$ & 2 & $5 \%$ \\
CU & 27 & $63 \%$ & 11 & $26 \%$ \\
DE & 12 & $28 \%$ & 9 & $21 \%$ \\
RE & 26 & $60 \%$ & 6 & $14 \%$ \\
SB & 6 & $14 \%$ & 2 & $5 \%$ \\
CR & 10 & $23 \%$ & 4 & $9 \%$ \\
BI & 6 & $14 \%$ & 2 & $5 \%$ \\
DI & 20 & $47 \%$ & 12 & $28 \%$ \\
IC & 22 & $51 \%$ & 9 & $21 \%$ \\
FD & 25 & $58 \%$ & 11 & $26 \%$ \\
HG & 16 & $37 \%$ & 6 & $14 \%$ \\
ICA & 1 & $2 \%$ & 0 & $0 \%$ \\
PP & 13 & $30 \%$ & 3 & $7 \%$ \\
LT & 14 & $33 \%$ & 4 & $9 \%$ \\
UN & 7 & $16 \%$ & 1 & $2 \%$ \\
& 222 & & 82 & \\
\hline
\end{tabular}

Citation: Luotolahti H, Kantola I,Viikari J.Weight loss has a rapid positive impact on the obese person's attitudes. Attitudes that could disturb weight loss attempts, decrease in number as the successful weight loss gets started. Adv Obes Weight Manag Control. 20I6;4(5):I33-I35. 
Table 3 Total number of appearances of factors before and after the successful weight loss period of eight weeks of VLCD

\begin{tabular}{lll}
\hline & Begining & End \\
\hline Total number of factors & 222 & 82 \\
Number of persons & 43 & 43
\end{tabular}

Table 4 The statistically significant changes in the amount of existing factors during the eight weeks of VLCD

\begin{tabular}{llll}
\hline Factor & Amount before VLCD & Amount after VLCD & Statistical Significance \\
\hline Inertia & $17 / 43(40 \%)$ & $2 / 43(5 \%)$ & $\mathrm{P}=0,002$, very statistically significant \\
Culinary attitude & $27 / 43(63 \%)$ & $\mathrm{I} / / 43(26 \%)$ & $\mathrm{P}=0,033$, statistically significant \\
Restlessness & $26 / 43(60 \%)$ & $6 / 43(14 \%)$ & $\mathrm{P}=0,003$, statistically significant \\
Lack of Time & $\mid 4 / 43(33 \%)$ & $4 / 43(9 \%)$ & $\mathrm{P}=0,038$, statistically significant \\
Peer pressure & $13 / 43(30 \%)$ & $3 / 43(7 \%)$ & $\mathrm{P}=0,028$, statistically significant \\
\hline
\end{tabular}

Statistical significance in the difference of amounts: $p=0.000$ I, extremely statistically significant. Fisher's exact test was used.

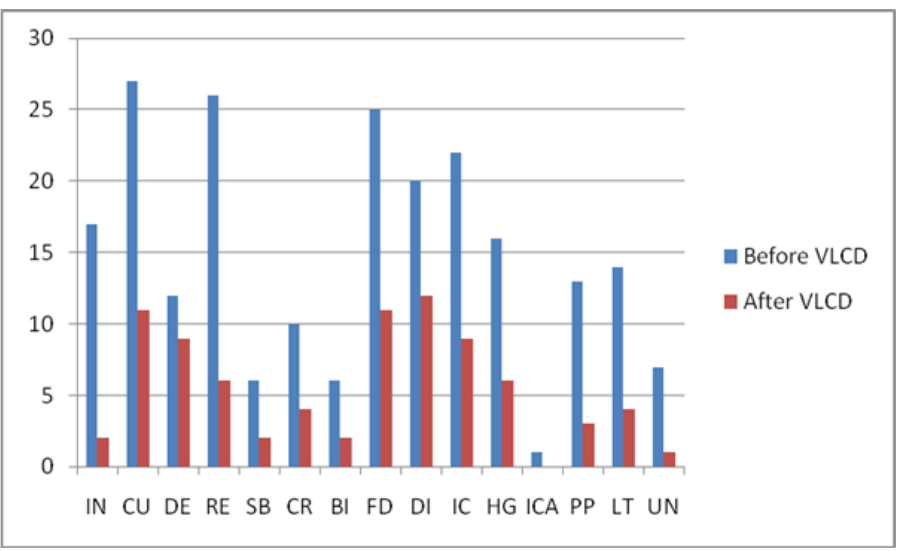

Figure I The appearance of the factors before and after the eight week of VLCD.

VLCD, very low calorie diet; IN, inertia; CU, culinary attitude; DE, depressive mood; RE, restlessness; SB, superiority attitude; CR, criticism; BI, bitterness; FD, food dependency; DI, experiencing general difficulties in weight loss; IC, impaired physical condition; HG, hunger; ICA, incapacity; PP, peer pressure; LT, lack of time; UN, unrealistic activity

\section{Limitations}

The questioning process is subjective and prone to misinterpretations. It is not known whether this is only a temporarily phenomenon that vanishes soon.

\section{Conclusion}

There seems to be found attitude-related factors that have a negative impact on the weight loss process. In of our study we found out, that these factors become less abundant as the successful weight loss happens. The total amount of factors decreased statistically extremely significantly as the successful weight loss happened.
These findings support the "Just do it"-approach when it comes to weight loss. Many difficulties felt in the beginning disappeared automatically as the weight loss started to happen. No psychological support was given during the diet period to get rid of these factors.

According to this study the encouragement to get started, and to keep going, seems to be more important than analyzing difficulties. According this study difficulties seem to lose their power as the successful weight loss happens. This change happens very fast, in weeks. Even the factors that one could assume being relatively permanent such as attitudes about having poor condition changed for the better.

It is possible that the weight loss itself is such a positive process, that it caused the decrease in difficult feelings and increased the capacity to handle obstacles of dieting. It pays to start the weight loss process in spite of disturbing factors. In spite of the limitations it might be hoped that the findings in this study could be used as a source for new ideas, study protocols and developments in the demanding field of weight loss management.

\section{Acknowledgements}

We warmly thank Hilda Kauhanen Memorial Fund, Pori, Finland for the most generous financial support for this study. The Study Protocol was approved by the Ethics Committee of the Hospital District of Southwest Finland. This study has been approved by an Institutional Review Board and it has the authorization of the hospital (number 35/180/2010, study accepted nr. 532/10).

\section{Conflict of interest}

The author declares no conflict of interest.

\section{References}

1. Luotolahti H, Viikari J, Kantola I. Attitudes that Make Weight Loss Management Difficult. Adv Obes Weight Manag Control. 2015;2(5):00031.

Citation: Luotolahti H, Kantola I,Viikari J.Weight loss has a rapid positive impact on the obese person's attitudes. Attitudes that could disturb weight loss attempts, decrease in number as the successful weight loss gets started. Adv Obes Weight Manag Control. 2016;4(5):I33-I35. 